\title{
Shorting out the cellular battery
}

\section{A low-resolution structure of the bacterial toxin colicin la provides a model for its lethal membrane association and an explanation for the anomalous behaviour of its C-terminal peptide fragment.}

Possibly the most significant single feature that distinguishes living cells from dead ones is their ability to establish and maintain an electrochemical potential across their plasma membranes. By pumping specific ions (in particular $\mathrm{Na}^{+}$and $\mathrm{K}^{+}$) across the cell membrane, a potential of -20 to $-200 \mathrm{mV}$ is produced, depending on organism and cell type. Without this potential the cell dies, a fact exploited by a number of toxins which kill their target cells by depleting this potential in an action exactly analogous to shorting out an electric battery. To achieve this, the toxin punches holes in the cell membrane causing ions to rush across and the potential to collapse.

Among the toxins that act in this fashion are a group of proteins produced by Escherichia coli known as the poreforming colicins. Colicins are encoded on a bacterial plasmid which also conveys resistance to colicin attack. A small proportion of plasmid-bearing cells will be producing colicin at any one time, destroying any bacteria not bearing the colicin plasmid and so clearing the way for those that do. In order to penetrate the complex bacterial cell wall these toxins undergo a series of structural changes, but until now little has been known of their structure and less still of their mechanism of action. Now with the first structure of an intact colicin, colicin Ia (ref. 1), albeit at relatively low resolution $(4 \AA)$, a unified picture of their bacteriolytic activity is beginning to emerge ${ }^{1,2}$

Ghosh and colleagues ${ }^{1}$ have succeeded in crystallizing the intact soluble form of colicin Ia and have calculated a $4 \AA$ electron density map. Although at this resolution it is not possible to follow the amino-acid chain through the protein, regular pillars of electron density indicate the positions of $\alpha$-helices. The locations of a few individual amino-acid residues could also be assigned, giving confidence in the accuracy of the electron density map. Colicin Ia is clearly seen to be composed of several $\alpha$-helices, linked by

Also in this month's Nature Structural Biology: detailed structures of a TATAbox-binding protein with and without bound DNA; an isolated $\beta$-hairpin free in solution; identifying the DNA-binding site of heat-shock transcription factor; and the structure of a pertussis toxin-sugar complex. irregular regions which cannot be seen at this resolution. The structure is divided into three domains arranged in a rough ' $\mathrm{Y}$ ' shape, $95 \AA$ long and $75 \AA$ across the two arms. By a synthesis of previous biochemical data and an earlier structure of a fragment from a related protein, colicin $\mathrm{A}$ (ref. 3), it can be shown that the two arms are formed by the carboxy and central two-thirds of the molecule while the 'stem' comprises the amino terminus. A separate function has been localized to each of these three domains.

The initial step in bacterial cell attack is attachment to the outer bacterial membrane by binding to receptors on the target cell's surface. This is a process common to many cellular toxins and the specific receptors are frequently carbohydrate moieties: see for example the structure of pertussis toxin bound to an undeccasaccharide in this month's Nature Structural Biology $y^{4}$. For colicin Ia this function is mapped to the central region of the protein which forms one of the arms of the ' $\mathrm{Y}$ ' (magenta in the figure).

After binding to its receptor, the toxin passes across the periplasmic space to reach the inner bacterial membrane. This translocation requires the $\mathrm{N}$-terminal portion of the molecule which forms the stem of the ' $Y$ ' and is made up of three $\alpha$ helices, the longest of which is about $70 \AA$. This means that colicin Ia may attach to the bacterial outer membrane by this $\mathrm{N}$-terminal bridging domain while the arms of the ' $Y$ ' stretch out to touch the inner cell membrane.

Finally, the carboxy-terminal third of the molecule (yellow and orange in the figure) inserts into the inner cell membrane, forming a nonspecific ion channel. This is a two-step reaction: two hydrophobic $\alpha$-helices insert into the membrane as a $\beta$-hairpin then, in the presence of an electrical potential, more helices are driven into the membrane to form a voltage-gated pore. This pore remains open until the membrane potential rises to around $+90 \mathrm{mV}$, a complete reversal of the normal situation.

C-terminal fragments of a number of colicins, including colicin Ia, can form ion channels in lipid membranes in vitro, but their physico-chemical properties (voltage dependence, open times and so on) are different from those of the intact protein. It is fragments of this kind whose structures have previously been determined ${ }^{3,5}$. These are composed of a

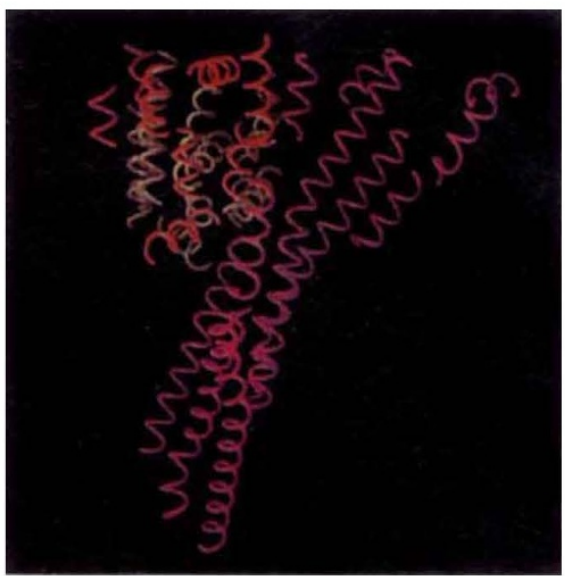

three-layered bundle of ten $\alpha$-helices formed around the two hydrophobic helices that initially insert into the membrane. In the complete molecule this fragment is seen as part of one arm of the ' $\mathrm{Y}$ ' (yellow), but there are also another eight $\alpha$-helices making up the rest of the 'arm' (orange). This subdomain may well be responsible for the differences in activity between $\mathrm{C}$-terminal pore-forming fragments and the intact colicins. Our models of membrane insertion, derived primarily from isolated pore-forming fragments, will now need to be recast.

As so often with solutions of protein structures, more questions have been raised than answered, answers that must come from a combination of different techniques. This structure also highlights the difficulty of interpreting data from protein fragments without knowing how such fragments fit into the whole. Now with our clearer picture of colicin Ia, and therefore of other homologous colicins, studies of its attachment, translocation and membrane insertion can proceed apace. This work on what is a relatively simple toxin can now be used to model the activity of more complex toxins, including the causative agents of diphtheria and cholera.

Christopher Surridge

Christopher Surridge is Assistant Editor of Nature Structural Biology.

\footnotetext{
1. Ghosh, P., Mel, S.F \& Stroud, R.M. Nature struct. Biol. 1, 597-604 (1994).

. Sansom, M., Nature struct. Biol. 1, 563-567 (1994).

3. Parker, M.W., Postma, J.P.M., Pattus, F., Tucker, A.D. \& Tsernoglou, D. J. molec. Biol. 224, 639-657 (1992). 4. Stein, P.E. et al. Nature struct. Biol. 1, 591-596 (1994).

5. Wormald, M.R., Merrill, A.R., Cramer, W.A. \& Williams,
} R.J.P. Eur. J. Biochem. 191, 155-161 (1990). 